\title{
Irreducibly represented groups
}

\author{
Bachir Bekka and Pierre de la Harpe*
}

\begin{abstract}
A group is irreducibly represented if it has a faithful irreducible unitary representation. For countable groups, a criterion for irreducible representability is given, which generalises a result obtained for finite groups by W. Gaschütz in 1954. In particular, torsionfree groups and infinite conjugacy class groups are irreducibly represented.

We indicate some consequences of this for operator algebras. In particular, we characterise up to isomorphism the countable subgroups $\Delta$ of the unitary group of a separable infinite dimensional Hilbert space $\mathscr{H}$ of which the bicommutants $\Delta^{\prime \prime}$ (in the sense of the theory of von Neumann algebras) coincide with the algebra of all bounded linear operators on $\mathscr{H}$.
\end{abstract}

Mathematics Subject Classification (2000). 22D10, $20 \mathrm{C} 07$.

Keywords. Group representations, irreducible representations, faithful representations, infinite groups, von Neumann algebras.

\section{Gaschütz Theorem for infinite groups, and consequences}

Define a group to be irreducibly represented if it has a faithful irreducible unitary representation and irreducibly underrepresented ${ }^{1}$ if not. For example, a finite abelian group is irreducibly represented if and only if it is cyclic (because finite subgroups of multiplicative groups of fields, in particular finite subgroups of $\mathbb{C}^{*}$, are cyclic). It is a straightforward consequence of Schur's lemma that a group of which the centre contains a non-cyclic finite subgroup is irreducibly underrepresented. For finite groups, there are also standard examples of groups without centre which are irreducibly underrepresented (see Note F in [Burns11]); moreover, there exists a criterion due to Gaschütz who states for finite dimensional representations over algebraically closed fields of characteristic zero the equivalence of Properties (i), (iv), and (v) in Theorem 2 below (see [Gasch54], as well as [Hupp98], § 42, and [Pálfy79]).

\footnotetext{
* The authors are grateful to the Swiss National Science Foundation for its support.

${ }^{1}$ On the day of writing, Google shows 29000000 entries for represented groups, 2390000 for underrepresented groups, 641000 for "represented groups", 670000 for "underrepresented groups", and zero entry for "irreducibly underrepresented groups". In some sense at least, what we have to say is new.
} 
The purpose of the present paper is to extend Gaschütz' result to infinite groups and unitary representations; for the particular case of finite groups, our arguments provide a new proof of the main result of [Gasch54] (at least for complex representations). For a generalisation of Gachütz' result of a rather different kind, see [Tushe93].

Since our arguments use measure theory, it is convenient to avoid the difficulties connected with non-standard spaces, so that we assume systematically that the groups involved are countable (see also Example VII in Subsection 5.1 below). Moreover and from now on, we write "representation" for "unitary representation" and, similarly, "character" for "unitary character".

To formulate our results, we need the following preliminaries. Let $\Gamma$ be a group.

Let $N$ be a normal subgroup of $\Gamma$. A representation $\sigma$ of $N$ is said to be $\Gamma$-faithful if $\bigcap_{\gamma \in \Gamma} \operatorname{ker}\left(\sigma^{\gamma}\right)=\{e\}$, where $e$ denotes the unit element of the group and where $\sigma^{\gamma}$ denotes the representation $n \mapsto \sigma\left(\gamma n \gamma^{-1}\right)$, namely the conjugate of $\sigma$ by $\gamma$. For example, if $\mathrm{V}$ denotes the normal subgroup of order 4 in the symmetric group Sym(4) on four letters, any character of V distinct from the unit character is Sym(4)-faithful (even though $\mathrm{V}$ does not have any faithful character).

If $\left\{S_{i}\right\}_{i \in I}$ is a family of subsets of $\Gamma$, we denote by $\left\langle\left\{S_{i}\right\}_{i \in I}\right\rangle$ the subgroup of $\Gamma$ generated by $\bigcup_{i \in I} S_{i}$. Following [Remak30], we define a foot of $\Gamma$ to be a minimal normal subgroup of $\Gamma$, namely a normal subgroup $M$ in $\Gamma$ such that $M \neq\{e\}$, and any normal subgroup of $\Gamma$ contained in $M$ is either $M$ or $\{e\}$. We denote by $\mathcal{F}_{\Gamma}$ the set of finite feet of $\Gamma$. The minisocle of $\Gamma$ is the subgroup $M S(\Gamma)$ of $\Gamma$ generated by the union of its finite feet; it is a characteristic subgroup of $\Gamma$. Let $\mathcal{A}_{\Gamma}$ denote the subset of $\mathcal{F}_{\Gamma}$ of abelian groups, and let $\mathscr{H}_{\Gamma}$ be the complement of $\mathcal{A}_{\Gamma}$ in $\mathcal{F}_{\Gamma}$. We define $M A(\Gamma)$ and $M H(\Gamma)$ to be the subgroups of $\Gamma$ generated by $\bigcup_{A \in \mathcal{A}_{\Gamma}} A$ and $\bigcup_{H \in \mathscr{H}_{\Gamma}} H$ respectively; both are characteristic subgroups of $\Gamma$ contained in $M S(\Gamma)$. By the usual convention, $M S(\Gamma)=\{e\}$ if $\mathcal{F}_{\Gamma}$ is empty, and similarly for $M A(\Gamma)$ and $M H(\Gamma)$.

Proposition 1. Let $\Gamma$ be a group, and let the notation be as above.

(i) Each $A \in \mathcal{A}_{\Gamma}$ is isomorphic to $\left(\mathbb{F}_{p}\right)^{n}$ for some prime $p$ and some positive integer $n$ (depending on $A$ ).

(ii) There exists a subset $\left\{A_{i}\right\}_{i \in I}$ of $\mathcal{A}_{\Gamma}$ such that $M A(\Gamma)=\bigoplus_{i \in I} A_{i}$. In particular, the group $M A(\Gamma)$ is abelian.

(iii) For each $H \in \mathscr{H}_{\Gamma}$, the feet $S_{1}, \ldots, S_{k}$ of $H$ are conjugate in $\Gamma$, and simple. Moreover $H=S_{1} \oplus \cdots \oplus S_{k}$.

(iv) We have $\operatorname{MH}(\Gamma)=\bigoplus_{H \in \mathscr{H}_{\Gamma}} H$.

(v) We have $\operatorname{MS}(\Gamma)=M A(\Gamma) \oplus M H(\Gamma)$.

For some examples of minisocles, see Section 5.1. Here is our first main result. 
Theorem 2. Let $\Gamma$ be a countable group. Let $M A(\Gamma)=\bigoplus_{i \in I} A_{i}$ and $M S(\Gamma)=$ $M A(\Gamma) \oplus M H(\Gamma)$ be as above. The following properties are equivalent:

(i) $\Gamma$ is irreducibly represented;

(ii) $M A(\Gamma)$ has a $\Gamma$-faithful character;

(iii) $M S(\Gamma)$ has a $\Gamma$-faithful irreducible representation;

(iv) for every finite subset $E$ of $I$, there exists an element $x_{E}$ in $M A_{E}(\Gamma) \doteqdot$ $\bigoplus_{i \in E} A_{i}$ such that the $\Gamma$-conjugacy class of $x_{E}$ generates $M A_{E}(\Gamma)$;

(v) for every pair of finite subsets $E \subset I$ and $F \subset \mathscr{H}_{\Gamma}$, there exists an element $z_{E, F}$ in $M S_{E, F}(\Gamma) \doteqdot\left(\bigoplus_{i \in E} A_{i}\right) \oplus\left(\bigoplus_{H \in F} H\right)$ such that the $\Gamma$-conjugacy class of $z_{E, F}$ generates $M S_{E, F}(\Gamma)$.

In particular, a countable group $\Gamma$ has a faithful irreducible representation as soon as $M A(\Gamma)=\{e\}$, and a fortiori as soon as $M S(\Gamma)=\{e\}$.

The next corollary is a straightforward consequence of Theorem 2. Recall that a group is icc if it is not reduced to one element and if all its conjugacy classes distinct from $\{e\}$ are infinite.

Corollary 3. For a countable group to be irreducibly represented, any of the three following conditions is sufficient: (i) the group is torsionfree, (ii) the group is icc, (iii) the group has a faithful primitive action on an infinite set.

The case of icc groups is well known, sometimes with a different proof. Indeed, a group is icc if and only if its von Neumann algebra is a factor of type $I I_{1}$ (Lemma 5.3.4 of [ROIV]); it is then a standard fact that the reduced $\mathrm{C}^{*}$-algebra of an icc group has a faithful irreducible representation, so that $a$ fortiori the group itself has a faithful irreducible representation (see for example Proposition 21 of [Harpe07]).

For a group $\Gamma$ which has a faithful primitive action on an infinite set $X$ (see [GelG108]), observe that any normal subgroup of $\Gamma$ not reduced to $\{e\}$ is transitive on $X$ and therefore infinite, so that $M S(\Gamma)=\{e\}$.

Theorem 2 does not state anything on the dimensions of the representations which can occur in (i). Before providing some information, let us recall that a group is virtually abelian if it has an abelian subgroup of finite index.

Theorem 4. For a countable group $\Gamma$, the two following properties are equivalent:

(i) $\Gamma$ has an infinite dimensional faithful irreducible representation;

(ii) $\Gamma$ has the properties of Theorem 2 and is not virtually abelian.

In other words, the following properties are equivalent:

(iii) $\Gamma$ has a faithful irreducible representation, and all its faithful irreducible representations are finite dimensional; 
(iv) $\Gamma$ has the properties of Theorem 2 and is virtually abelian.

Let $M$ be a von Neumann algebra. We denote by $U(M)$ the unitary group $\left\{X \in M \mid X^{*} X=X X^{*}=1\right\}$ of $M$ and by $S^{\prime \prime}$ the double commutant of a subset $S$ of $M$. Recall that $M$ is a factor if its centre is reduced to $\mathbb{C}$, a factor of type $I$ if there exists a Hilbert space $\mathscr{H}$ such that $M=\mathscr{L}(\mathscr{H})$, and a factor of type $I_{\infty}$ in case $\mathscr{H}$ is infinite dimensional (moreover, we assume here that Hilbert spaces are separable). For factors of type I, we write $U(\mathscr{H})$ instead of $U(M)$.

Corollary 5. Let $M=\mathscr{L}(\mathscr{H})$ be a factor of type $I_{\infty}$. For a countable group $\Gamma$, the following two properties are equivalent:

- there is a subgroup $\Delta$ of $U(\mathscr{H})$ isomorphic to $\Gamma$ such that $\Delta^{\prime \prime}=M$;

- $\Gamma$ has the properties of Theorem 2 and is not virtually abelian.

It would be interesting to have some information of this kind for other factors. In particular, we do not know any analogue of Theorem 4 for any given finite dimension $n \geq 2$, nor of Corollary 5 for the finite dimensional factor $\mathscr{L}\left(\mathbb{C}^{n}\right)$. We do not know any solution to the a priori easier problem to characterise the countable groups which have at least one finite dimensional faithful irreducible representation.

The proof of Proposition 1 uses standard arguments (compare with Section 4.3 of [DixMo96]). For the convenience of the reader, we give details in Section 2. Theorem 2 is proved in Section 3. Theorem 4 and Corollary 5 are proved in Section 4. We formulate a few remarks in Section 5: on examples of socles and minisocles, on the comparison between minisocles and periodic FC-kernels, on a theorem of Gelfand and Raikov, on tensor products of faithful representations, and on countable groups with primitive maximal $\mathrm{C}^{*}$-algebras. The final Section 6 is devoted to a generalisation of Theorem 2 concerning a countable group $\Gamma$ given together with a group of automorphisms $G$ which contains the group of inner automorphisms.

Understanding groups of a given class includes understanding their faithful actions of various kinds, and the setting of linear (or unitary) actions is only one among several others. For example, in the case of finite groups, the questions of classifying multiply transitive actions and primitive actions which are faithful have been central in group theory for more than hundred years; faithful primitive actions for infinite groups have been addressed in [GelGl08]. Faithful amenable actions are the subject of [GlaMo07]. Our initial motivation has been to ask some of the corresponding questions for linear actions.

We are most grateful to Yair Glasner for explaining us his work [GelGl08] and for his contribution to the setting out of the present work, to Yehuda Shalom for a useful observation, and to Yves de Cornulier and John Wilson for their remarks on a preliminary version of this paper. 


\section{Proof of Proposition 1}

We prepare the proof of Proposition 1 by recalling two lemmas.

Lemma 6. Let $\Gamma$ be a group. Let $M$ be a minimal normal subgroup of $\Gamma$ and $N$ a normal subgroup of $\Gamma$. Then either $M \subset N$ or $\langle M, N\rangle=M \oplus N$.

Proof. We can assume $N \neq\{e\}$. Since $M \cap N$ is both in $M$ and normal in $\Gamma$, either $M \cap N=M$, and $M \subset N$, or $M \cap N=\{e\}$, and $\langle M, N\rangle=M \oplus N$.

Lemma 7. Let $A$ be a group and let $\left(S_{i}\right)_{i \in I}$ be a family of nonabelian simple groups; set $S=A \oplus\left(\bigoplus_{i \in I} S_{i}\right)$. Let $M$ be a minimal normal subgroup of $S$.

Then either $M=S_{\ell}$ for some $\ell \in I$, or $M \subset A$.

Proof. Assume that $M \neq S_{\ell}$ for all $\ell \in I$. Choose $i \in I$; by Lemma 6 applied to $M$ and $N=S_{i}$, the groups $M$ and $S_{i}$ commute. It follows that $M$ is a subgroup of the centraliser of $\bigoplus_{i \in I} S_{i}$ in $S$, namely a subgroup of $A$.

Proof of Proposition 1. (i) Let $A \in \mathcal{A}_{\Gamma}$. By the structure theory of finite abelian groups, there exist a prime $p$ and an element $a \in A$ of order $p$. Let $A^{*}$ denote the set of elements of order $p$ in $A$. Then $A^{*} \cup\{e\}$ is a characteristic subgroup of $A$, and therefore a normal subgroup of $\Gamma$. By minimality of $A$, we have $A^{*} \cup\{e\}=A$, so that $A$ is isomorphic to $\left(\mathbb{F}_{p}\right)^{n}$ for some $n \geq 1$, as claimed.

(ii) Let $\mathscr{L}$ be the set of subsets $\left\{A_{\ell}\right\}_{\ell \in L}$ of $\mathcal{A}_{\Gamma}$ such that $\left\langle\left\{A_{\ell}\right\}_{\ell \in L}\right\rangle=\bigoplus_{\ell \in L} A_{\ell}$; we order $\mathscr{L}$ by inclusion. The crucial observation is that the ordered set $\mathscr{L}$ is inductive, so that we can choose a maximal element, say $\left\{A_{i}\right\}_{i \in I}$. Suppose that $\bigoplus_{i \in I} A_{i}$ is strictly contained in $M A(\Gamma)$; we will arrive at a contradiction.

Choose $B \in \mathcal{A}_{\Gamma}$ such that $B$ is not contained in $\bigoplus_{i \in I} A_{i}$. By Lemma 6 applied to $M=B$ and $N=\bigoplus_{i \in I} A_{i}$, we have either $B \subset \bigoplus_{i \in I} A_{i}$, which is ruled out by the choice of $B$, or $\left\langle B,\left\{A_{i}\right\}_{i \in I}\right\rangle=B \oplus\left(\bigoplus_{i \in I} A_{i}\right)$, which is ruled out by the maximality of $I$. This is the announced contradiction.

(iii) Let $H \in \mathscr{H}_{\Gamma}$. Choose a minimal normal subgroup $S$ in $H$ (this is possible since $H$ is finite). For each $x \in \Gamma$, the subgroup $x S x^{-1}$ is minimal normal in $H$. Choose a set $S_{1}, \ldots, S_{k}$ of such conjugates of $S$ in $\Gamma$ which is such that $\left\langle S_{1}, \ldots, S_{k}\right\rangle=S_{1} \oplus \cdots \oplus S_{k}$ and which is maximal for this property. Set $N=\left\langle S_{1}, \ldots, S_{k}\right\rangle$; it is a normal subgroup of $H$.

We claim that $x S s^{-1} \subset N$ for each $x \in \Gamma$, so that $N$ is normal in $\Gamma$. Indeed, by Lemma 6 applied to $M=x S x^{-1}$ and $N$ in $H$, either $\left\langle x S x^{-1}, S_{1}, \ldots, S_{k}\right\rangle=$ $x S x^{-1} \oplus S_{1} \oplus \cdots \oplus S_{k}$, but this is ruled out by the maximality of the set $\left\{S_{1}, \ldots, S_{k}\right\}$, or $x S x^{-1} \subset N$, and this establishes the claim.

Since $N$ is normal in $\Gamma$ and $N \subset H$, we have $N=H$ by minimality of $H$. Observe that, for each $i \in\{1, \ldots, k\}$, any normal subgroup of $S_{i}$ is normal in $H$; it 
follows that $S_{i}$ is a simple group. Finally, the set $\left\{S_{1}, \ldots, S_{k}\right\}$ coincides with the set of all minimal normal subgroups of $H$ by Lemma 7 .

(iv) The same argument as for (ii) shows that there exists a subset $\left\{H_{k}\right\}_{k \in K}$ of $\mathscr{H}_{\Gamma}$ such that $\bigoplus_{k \in K} H_{k}=M H(\Gamma)$, and Lemma 7 implies that $\left\{H_{k}\right\}_{k \in K}=\mathscr{H}_{\Gamma}$.

(v) Again by the same argument as for (ii), there exists a subset $\left\{M_{\ell}\right\}_{\ell \in L}$ of $\mathcal{F}_{\Gamma}$ such that $\bigoplus_{\ell \in L} M_{\ell}=M S(\Gamma)$, and Lemma 7 implies that $\left\{M_{\ell}\right\}_{\ell \in L}$ contains $\mathscr{H}_{\Gamma}$.

\section{Proof of Theorem 2}

We will prove successively that

$$
\begin{array}{rlll}
\text { (i) } & \Longrightarrow & \text { (ii) \& (iii) } & \text { (see Lemma 9), } \\
\text { (iii) } & \Longrightarrow & \text { (i) } & \text { (Lemma 10), } \\
\text { (ii) } & \Longleftrightarrow & \text { (iii) } & \text { (Lemma 13), } \\
\text { (ii) } & \Longleftrightarrow \text { (iv) } & \text { (Lemma 14). }
\end{array}
$$

The equivalence (iv) $\Longleftrightarrow$ (v) is straightforward, since nonabelian feet are direct products of simple groups. Recall that we write "representation" for "unitary representation".

Given a representation $\pi$ of a countable group $\Gamma$ in a Hilbert space $\mathcal{H}$, there exist a standard Borel space $\Omega$, a bounded positive measure $\mu$ on $\Omega$, a measurable field $\omega \mapsto \pi_{\omega}$ of irreducible representations of $\Gamma$ in a measurable field $\omega \mapsto \mathscr{H}_{\omega}$ of Hilbert spaces on $\Omega$, and an isomorphism of $\mathscr{H}$ with $\int_{\Omega}^{\oplus} \mathscr{H}_{\omega} d \mu(\omega)$ which implements a unitary equivalence

$$
\pi(\gamma) \approx \int_{\Omega}^{\oplus} \pi_{\omega}(\gamma) d \mu(\omega)
$$

for all $\gamma \in \Gamma$. See [Dix69C*, Sections 8.5 and 18.7.6]. (Such decompositions in irreducible representations carry over to continuous representations of separable locally compact groups, and more generally of separable $\mathrm{C}^{*}$-algebras. They are applications of the reduction theory for von Neumann algebras [Dix69vN, Chapter II]). The following lemma is standard, but we haven't found any appropriate reference.

Lemma 8. Let $\Gamma$ be a countable group. Let $\Omega$ be a measure space with a positive measure $\mu$. Let $\omega \mapsto \pi_{\omega}$ be a measurable field of representations of $\Gamma$ in a measurable field of Hilbert spaces $\omega \mapsto \mathscr{H}_{\omega}$ over $\Omega$ and let $\gamma \in \Gamma$.

Then $\left\{\omega \in \Omega \mid \pi_{\omega}(\gamma)=I\right\}$ is a measurable subset of $\Omega$.

Proof. Let $\left(\xi^{(1)}, \xi^{(2)}, \ldots\right)$ be a fundamental sequence of measurable vector fields (see [Dix69vN, Chapter II, Number 1.3]). For $i, j \geq 1$, consider the set

$$
\Omega_{i, j}=\left\{\omega \in \Omega \mid\left\langle\pi_{\omega}(\gamma) \xi^{(i)}(\omega), \xi^{(j)}(\omega)\right\rangle=\left\langle\xi^{(i)}(\omega), \xi^{(j)}(\omega)\right\rangle\right\} .
$$


Observe that

$$
\left\{\omega \in \Omega \mid \pi_{\omega}(\gamma)=I\right\}=\bigcap_{i, j \geq 1} \Omega_{i, j} .
$$

Therefore it suffices to show that each set $\Omega_{i, j}$ is measurable.

For fixed $i, j \geq 1$, the functions

$$
\omega \longmapsto\left\langle\xi^{(i)}(\omega), \xi^{(j)}(\omega)\right\rangle \text { and } \omega \longmapsto\left\langle\pi_{\omega}(\gamma) \xi^{(i)}(\omega), \xi^{(j)}(\omega)\right\rangle
$$

are measurable, by definition of a measurable vector field and of a measurable field of representations. Hence $\Omega_{i, j}$ is measurable and the proof is complete.

Let us now recall a general fact which can be seen as a weak form of Clifford theorem for infinite dimensional representations. (For a version of Clifford theorem concerning finite dimensional representations but possibly infinite groups, see Theorem 2.2 in [Dixon71].)

Lemma 9. Let $\Gamma$ be a countable group, $N$ a normal subgroup, $\pi$ an irreducible representation of $\Gamma$ in a Hilbert space $\mathscr{H}$, and $\sigma$ the restriction of $\pi$ to $N$. Identify $\sigma$ to a direct integral of irreducible representations

$$
\sigma=\left.\pi\right|_{N}=\int_{\Omega}^{\oplus} \sigma_{\omega} d \mu(\omega)
$$

as above.

If the representation $\pi$ is faithful, then the representation $\sigma_{\omega}$ is $\Gamma$-faithful for almost all $\omega \in \Omega$.

Proof. If $N=\{e\}$, there is nothing to prove. We assume from now on that $N$ is not reduced to one element.

Denote by $\left\{C_{j}\right\}_{j \in J}$ the family of $\Gamma$-conjugacy classes in $N$ distinct from $\{e\}$. For each $j \in J$, denote by $N_{j}$ the subgroup of $N$ generated by $C_{j}$; observe that each $N_{j}$ is normal in $\Gamma$, and that the family $\left\{N_{j}\right\}_{j \in J}$ is countable (possibly finite) and nonempty. Set

$$
\Omega_{j}=\left\{\omega \in \Omega \mid N_{j} \subset \operatorname{ker}\left(\bigoplus_{\gamma \in \Gamma} \sigma_{\omega}^{\gamma}\right)\right\} \quad \text { and } \quad \tilde{\Omega}=\bigcup_{j \in J} \Omega_{j}
$$

For $\omega \in \Omega$, observe that $\sigma_{\omega}$ is not $\Gamma$-faithful if and only if the kernel of $\bigoplus_{\gamma \in \Gamma} \sigma_{\omega}^{\gamma}$ contains one of the $N_{j}$; thus $\widetilde{\Omega}$ is the subset of $\Omega$ of the points $\omega$ such that $\sigma_{\omega}$ is not $\Gamma$-faithful. Each $\Omega_{j}$ is measurable in $\Omega$ as a consequence of Lemma 8; as $J$ is countable, $\widetilde{\Omega}$ is also measurable.

To end the proof, we assume that $\mu(\widetilde{\Omega})>0$ and we will arrive at a contradiction. 
As the family $J$ is countable, there exists $\ell \in J$ such that $\mu\left(\Omega_{\ell}\right)>0$. Hence the unit representation $1_{N_{\ell}}$ of the group $N_{\ell}$ is strongly contained in the restriction of $\sigma$ to $N_{\ell}$, so that the subspace of $\mathscr{H}$ of $N_{\ell}$-invariant vectors is not reduced to $\{0\}$. Since $N_{\ell}$ is normal in $\Gamma$, this subspace is invariant by $\pi(\Gamma)$; by irreducibility of $\pi$, this subspace is the whole of $\mathscr{H}$. In other words, the restriction of $\pi$ to $N_{\ell}$ is the unit representation. The last statement is a contradiction, since $\pi$ is faithful.

The particular case of Lemma 9 for which $N=M A(\Gamma)$ [respectively $N=$ $M S(\Gamma)$ ] shows that (i) implies (ii) [respectively (iii)] in Theorem 2. The implication (iii) $\Longrightarrow$ (i) follows from the next lemma applied to $N=M S(\Gamma)$ since, by definition, there does not exist any finite foot $M$ of $\Gamma$ such that $M \cap M S(\Gamma)=\{e\}$.

Lemma 10. Let $\Gamma$ be a countable group, $N$ a normal subgroup, $\sigma$ an irreducible representation of $N$ in a Hilbert space $\mathcal{K}$, and $\pi=\operatorname{Ind}_{N}^{\Gamma}(\sigma)$ the corresponding induced representation. Let $\pi=\int_{\Omega}^{\oplus} \pi_{\omega} d \mu(\omega)$ be a direct integral decomposition of $\pi$ into irreducible representations. Assume that there does not exist any finite foot $M$ in $\Gamma$ such that $M \cap N=\{e\}$.

If the representation $\sigma$ is $\Gamma$-faithful, then the representation $\pi_{\omega}$ is faithful for almost all $\omega$ in $\Omega$.

Proof. In the model we choose for induced representations, $\pi$ acts on the Hilbert space $\mathscr{H}$ of mappings $f: \Gamma \rightarrow \mathcal{K}$ with the two following properties:

$$
\begin{aligned}
& \text { (1) } f(\gamma n)=\sigma\left(n^{-1}\right) f(\gamma) \quad \text { for all } \gamma \in \Gamma \text { and } n \in N, \\
& \text { (2) } \sum_{\Gamma / N}\|f(\gamma)\|^{2}<\infty
\end{aligned}
$$

(The notation of (2) indicates a summation over one representative $\gamma \in \Gamma$ of each class in $\Gamma / N$.) Then $(\pi(x) f)(\gamma)=f\left(x^{-1} \gamma\right)$ for all $x, \gamma \in \Gamma$.

Denote this time by $\left\{C_{j}\right\}_{j \in J}$ the family of conjugacy classes of $\Gamma$ distinct from $\{e\}$. For each $j \in J$, denote by $\Gamma_{j}$ the subgroup generated by $C_{j}$, which is a normal subgroup of $\Gamma$ not reduced to $\{e\}$; set

$$
\Omega_{j}=\left\{\omega \in \Omega \mid \Gamma_{j} \subset \operatorname{ker}\left(\pi_{\omega}\right)\right\} \quad \text { and } \quad \widetilde{\Omega}=\bigcup_{j \in J} \Omega_{j}
$$

As in the proof of Lemma $9, \widetilde{\Omega}$ is the set of points $\omega$ such that $\pi_{\omega}$ is not faithful, and it is measurable. To end the proof, we assume that $\mu(\widetilde{\Omega})>0$, so that there exists $\ell \in J$ for which $\mu\left(\Omega_{\ell}\right)>0$, and we will arrive at a contradiction.

Continuing as in the proof of Lemma 9, we observe that there exists a nonzero vector $f: \Gamma \rightarrow \mathcal{K}$ in $\mathscr{H}=\int_{\Omega}^{\oplus} \mathscr{H}_{\omega} d \mu(\omega)$ which is supported in $\Omega_{\ell}$ (as a measurable 
section of the field of Hilbert spaces $\omega \mapsto \mathscr{H}_{\omega}$ underlying the field of representations $\left.\omega \mapsto \pi_{\omega}\right)$, and which is such that $\pi(x) f=f$ for all $x \in \Gamma_{\ell}$.

Let $\gamma_{0} \in \Gamma$ be such that $f\left(\gamma_{0}^{-1}\right) \neq 0$; set $\xi=f\left(\gamma_{0}^{-1}\right)$. Using (1), we find

(3) $\xi=f\left(\gamma_{0}^{-1}\right)=f\left(x^{-1} \gamma_{0}^{-1}\right)=f\left(\gamma_{0}^{-1}\left(\gamma_{0} x^{-1} \gamma_{0}^{-1}\right)\right)=\sigma\left(\gamma_{0} x \gamma_{0}^{-1}\right) \xi=\sigma^{\gamma_{0}}(x) \xi$

for all $x \in \Gamma_{\ell} \cap N$.

Claim $1 . \Gamma_{\ell} \cap N=\{e\}$. Denote by $\mathcal{K}^{\Gamma_{\ell} \cap N}$ the subspace of $\mathcal{K}$ of vectors invariant by $\sigma^{\gamma_{0}}\left(\Gamma_{\ell} \cap N\right)$. This is a $\sigma^{\gamma_{0}}(N)$-invariant subspace of $\mathcal{K}$, since $\Gamma_{\ell} \cap N$ is a normal subgroup of $N$. Now $\mathcal{K}^{\Gamma_{\ell} \cap N} \neq\{0\}$ by (3) and $\mathcal{K}^{\Gamma_{\ell} \cap N}=\mathcal{K}$ because $\sigma^{\gamma_{0}}$ is irreducible. Thus $\Gamma_{\ell} \cap N$ is inside the kernel of the representation $\sigma^{\gamma_{0}}$ of $N$; as $\Gamma_{\ell} \cap N$ is normal in $\Gamma$, the group $\Gamma_{\ell} \cap N$ is also inside the kernel of the representation $\sigma^{\gamma}$ of $N$ for all $\gamma \in \Gamma$. As $\sigma$ is $\Gamma$-faithful, $\Gamma_{\ell} \cap N=\{e\}$, as claimed.

Claim 2. The subgroup $\Gamma_{\ell}$ of $\Gamma$ is finite. Consider the function

$$
\varphi: \Gamma \longrightarrow \mathbb{R}_{+}, \quad \gamma \longmapsto\|f(\gamma)\|
$$

We have

(4) $\varphi\left(\gamma_{0}^{-1}\right) \neq 0$,

(5) $\varphi$ is constant under right translations by elements of $N$,

(6) $\sum_{\Gamma / N}|\varphi(\gamma)|^{2}<\infty$

(7) $\varphi$ is invariant under left translations by elements of $\Gamma_{\ell}$.

It follows from (4) to (7) that the image of $\Gamma_{\ell} \gamma_{0}$ in $\Gamma / N$ is finite. The image of $\gamma_{0}^{-1} \Gamma_{\ell} \gamma_{0}=\Gamma_{\ell}$ in $\Gamma / N$ is also finite, so that the index of $N$ in $\Gamma_{\ell} N$ is finite. Claim 2 follows since $\Gamma_{\ell} N$ is isomorphic to the direct sum $\Gamma_{\ell} \oplus N$ by Claim 1.

Any subgroup $M$ of $\Gamma_{\ell}$ which is normal in $\Gamma$ and minimal for this property is a finite foot of $\Gamma$, and $M \cap N=\{e\}$ by Claim 1. This is in contradiction with one of the hypotheses of the lemma.

The particular case $N=\{e\}$ is of independent interest.

Proposition 11. Let $\Gamma$ be a countable infinite group which does not contain any finite foot, and let $\lambda_{\Gamma}=\int_{\Omega}^{\oplus} \pi_{\omega} d \mu(\omega)$ be a direct integral decomposition of the left regular representation $\lambda_{\Gamma}$ into irreducible representations. Then $\pi_{\omega}$ is faithful for almost all $\omega \in \Omega$.

Next, we show that (ii) $\Longleftrightarrow$ (iii) in Theorem 2. This will be a consequence of Lemma 13, for the proof of which we will call upon the following lemma.

For a Hilbert space $\mathscr{H}$, we denote by $\mathscr{L}(\mathscr{H})$ its algebra of bounded linear operators. 
Lemma 12. Let $\mathscr{H}_{1}, \mathscr{H}_{2}$ be two Hilbert spaces. Let $S_{1} \in \mathscr{L}\left(\mathscr{H}_{1}\right), S_{2} \in \mathscr{L}\left(\mathscr{H}_{2}\right)$ be such that $S_{1} \otimes S_{2} \in \mathscr{L}\left(\mathscr{H}_{1} \otimes \mathscr{H}_{2}\right)$ is a non-zero multiple of the identity operator.

Then $S_{1}$ and $S_{2}$ are multiples of the identity.

Proof. Let $\lambda \in \mathbb{C}^{*}$ be such that $S_{1} \otimes S_{2}=\lambda I$. Let $\left\{\xi_{i}\right\}_{i \in I}$ be a Hilbert space basis of $\mathscr{H}_{1}$. Since $S_{2} \neq 0$, there exist $\eta_{1}, \eta_{2} \in \mathscr{H}_{2}$ such that

$$
\left\langle S_{2}\left(\eta_{1}\right), \eta_{2}\right\rangle \neq 0
$$

For every $\xi \in \mathscr{H}_{1}$, we have

$$
\left\langle\left(S_{1} \otimes S_{2}\right)\left(\xi \otimes \eta_{1}\right), \xi_{i} \otimes \eta_{2}\right\rangle=\left\langle S_{1}(\xi), \xi_{i}\right\rangle\left\langle S_{2}\left(\eta_{1}\right), \eta_{2}\right\rangle
$$

and hence

$$
\begin{aligned}
\left\langle S_{1}(\xi), \xi_{i}\right\rangle & =\frac{1}{\left\langle S_{2}\left(\eta_{1}\right), \eta_{2}\right\rangle}\left\langle\left(S_{1} \otimes S_{2}\right)\left(\xi \otimes \eta_{1}\right), \xi_{i} \otimes \eta_{2}\right\rangle \\
& =\frac{\lambda}{\left\langle S_{2}\left(\eta_{1}\right), \eta_{2}\right\rangle}\left\langle\xi \otimes \eta_{1}, \xi_{i} \otimes \eta_{2}\right\rangle \\
& =\frac{\lambda\left\langle\eta_{1}, \eta_{2}\right\rangle}{\left\langle S_{2}\left(\eta_{1}\right), \eta_{2}\right\rangle}\left\langle\xi, \xi_{i}\right\rangle
\end{aligned}
$$

for all $i \in I$. It follows that

$$
\begin{aligned}
S_{1}(\xi) & =\sum_{i \in I}\left\langle S_{1}(\xi), \xi_{i}\right\rangle \xi_{i} \\
& =\frac{\lambda\left\langle\eta_{1}, \eta_{2}\right\rangle}{\left\langle S_{2}\left(\eta_{1}\right), \eta_{2}\right\rangle} \sum_{i \in I}\left\langle\xi, \xi_{i}\right\rangle \xi_{i} \\
& =\frac{\lambda\left\langle\eta_{1}, \eta_{2}\right\rangle}{\left\langle S_{2}\left(\eta_{1}\right), \eta_{2}\right\rangle} \xi
\end{aligned}
$$

for every $\xi \in H_{1}$, showing that $S_{1}$ is a multiple of the identity. A similar argument applies to $S_{2}$.

Lemma 13. Let $\Gamma$ be a group and let $N$ be a normal subgroup of $\Gamma$. Assume that $N=A \oplus S$, where $A$ is an abelian normal subgroup of $\Gamma$ and where $S$ is the direct sum of a family $\left(S_{i}\right)_{i \in I}$ of finite simple nonabelian normal subgroups of $S$. The following properties are equivalent:

(i) $N$ has a $\Gamma$-faithful irreducible representation;

(ii) A has a $\Gamma$-faithful character. 
Proof. Assume first that there exists a $\Gamma$-faithful irreducible representation $\pi$ of $N$. Since the factor $A$ of $N=A \oplus S$ is abelian, and in particular a type I group, there exist a character $\chi$ of $A$ and an irreducible representation $\rho$ of $S$ such that $\pi=\chi \otimes \rho$ [Dix69C*, Proposition 13.1.8]. Since $\operatorname{ker}\left(\chi^{\gamma}\right)=\operatorname{ker}\left(\left.\left(\pi^{\gamma}\right)\right|_{A}\right)$ for all $\gamma \in \Gamma$, the character $\chi$ of $A$ is $\Gamma$-faithful.

Assume now that there exists a $\Gamma$-faithful character $\chi$ of $A$. We claim that there exists an irreducible representation $\rho$ of $S$ such that, for every $\gamma \in S, \gamma \neq e$, the operator $\rho(\gamma)$ is not a multiple of the identity operator. Lemma 12 will then imply that the exterior tensor product $\chi \otimes \rho$ is a $\Gamma$-faithful representation of $N=A \oplus S$.

For every $i \in I$, let $\rho_{i}$ be an irreducible representation of $S_{i}$ distinct from the unit representation, in some Hilbert space $\mathscr{H}_{i}$. Choose a unit vector $\eta_{i} \in \mathscr{H}_{i}$. Consider the infinite tensor product $\rho=\bigotimes_{i \in I} \rho_{i}$ of the family $\left(\rho_{i}\right)_{i \in I}$ with respect to the family $\left(\eta_{i}\right)_{i \in I}$. Recall that $\rho$ is the representation of $S$ defined on the infinite tensor product $\mathscr{H}=\bigotimes_{i \in I}\left(\mathscr{H}_{i}, \eta_{i}\right)$ of the family of Hilbert spaces $\left(\mathscr{H}_{i}\right)_{i \in I}$ with respect to the family $\left(\eta_{i}\right)_{i \in I}$ by

$$
\rho\left(\left(\gamma_{i}\right)_{i \in I}\right)\left(\left(\bigotimes_{f \in F} \xi_{f}\right) \otimes\left(\bigotimes_{i \in I \backslash F} \eta_{i}\right)\right)=\left(\bigotimes_{f \in F} \rho_{i}\left(\gamma_{i}\right) \xi_{f}\right) \otimes\left(\bigotimes_{i \in I \backslash F} \eta_{i}\right),
$$

for every finite subset $F$ of $I$, element $\left(\gamma_{i}\right)_{i \in I} \in S$ with $\gamma_{i}=1$ whenever $i \in I \backslash F$, and decomposable vector $\left(\xi_{f}\right)_{f \in F} \in \bigotimes_{f \in F} \mathscr{H}_{f}$. The representation $\rho$ is irreducible, since the $\rho_{i}$ 's are irreducible. For all this, see for example [Guich66], in particular Corollary 2.1.

Let us check that, for $\gamma=\left(\gamma_{i}\right)_{i \in I} \in S, \gamma \neq e$, the operator $\rho(\gamma)$ is not a multiple of the identity operator. Choose $j \in I$ such that $\gamma_{j} \neq e$. Observe that the set

$$
\left\{\delta \in S_{j}: \rho_{j}(\delta) \text { is a multiple of the identity operator }\right\}
$$

is an abelian normal subgroup of $S_{j}$ and is therefore reduced to $\{e\}$ since $S_{j}$ is simple and nonabelian. The operator $\rho_{j}\left(\gamma_{j}\right)$ is therefore not a multiple of the identity operator. Denote by $\rho_{j}^{\prime}$ the tensor product of the family $\left(\rho_{\ell}\right)_{\ell \in I \backslash\{j\}}$, defined on $\mathscr{H}_{j}^{\prime}=\bigotimes_{\ell \in I \backslash\{j\}}\left(\mathcal{H}_{\ell}, \eta_{\ell}\right)$. We can then write

$$
\mathscr{H}=\mathscr{H}_{j} \otimes \mathscr{H}_{j}^{\prime} \quad \text { and } \quad \rho=\rho_{j} \otimes \rho_{j}^{\prime} .
$$

Lemma 12 implies that $\rho(\gamma)$ is not a multiple of the identity operator.

It remains to show that (ii) $\Longleftrightarrow$ (iv) in Theorem 2. This will be a consequence of the following lemma.

We are most grateful to Roland Lötscher, who pointed out a mistake at this point in a first version of our paper; we are also grateful to Jacques Thévenaz for a helpful discussion on modular representations. 
Lemma 14. Let $\Gamma$ be a countable group; set $A=M A(\Gamma)$. Let $\left\{A_{i}\right\}_{i \in I}$ be a set of finite abelian feet of $\Gamma$ as in Proposition 1, so that $A=\bigoplus_{i \in I} A_{i}$. For each finite subset $E$ of $I$, set $A_{E}=\bigoplus_{i \in E} A_{i}$, which is a finite abelian group. Let $\widehat{A}, \widehat{A}_{E}$ denote the dual group of $A, A_{E}$ respectively. The following properties are equivalent:

(i) A has a $\Gamma$-faithful character;

(ii) there exists a character $\chi \in \hat{A}$ such that the subgroup generated by $\chi^{\Gamma} \doteqdot\left\{\chi^{\gamma} \mid\right.$ $\gamma \in \Gamma\}$ is dense in $\widehat{A}$;

(iii) for every finite subset $E$ of $I$, the finite group $\widehat{A}_{E}$ has a $\Gamma$-faithful character;

(iv) for every finite subset $E$ of I, there exists $\chi \in \widehat{A}_{E}$ such that $\widehat{A}_{E}$ is generated by the $\Gamma$-orbit of $\chi$;

(v) for every finite subset $E$ of $I$, there exists $x_{E} \in A_{E}$ such that $A_{E}$ is generated by the $\Gamma$-conjugacy class of $x_{E}$.

Proof. Equivalence of (i) and (ii) and equivalence of (iii) and (iv). Let $N$ be a normal abelian subgroup of $\Gamma$. Let $\chi \in \hat{N}$. Denote by $H$ the closed subgroup of $\widehat{N}$ generated by $\chi^{\Gamma}$. By Pontrjagin duality, the unitary dual of the compact abelian group $\widehat{N} / H$ can be identified with the subgroup

$$
H^{\perp}=\{a \in N: \psi(a)=1 \text { for all } \psi \in H\} ;
$$

observe that

$$
H^{\perp}=\left\{a \in N: \psi(a)=1 \text { for all } \psi \in \chi^{\Gamma}\right\}=\bigcap_{\gamma \in \Gamma} \operatorname{ker}\left(\chi^{\gamma}\right) .
$$

Thus $\chi^{\Gamma}$ is dense in $\widehat{N}$ if and only if $H^{\perp}=\{e\}$, namely if and only if $\chi$ is $\Gamma$-faithful.

Equivalence of (ii) and (iii). It is clear that (ii) implies (iii). Let us assume that (iii) holds; we have to check that this implies (ii). For every finite subset $E$ of $I$, denote by $p_{E}: \hat{A} \rightarrow \widehat{A}_{E}$ the canonical projection. Consider the subset

$$
X_{E}=\left\{\chi \in \hat{A} \mid \text { the } \Gamma \text {-orbit of } p_{E}(\chi) \text { generates } \widehat{A}_{E}\right\} \text {. }
$$

Since the group $\hat{A}_{E}$ is finite, the subset $X_{E}$ of $\hat{A}$ is closed. For a finite family $E_{1}, \ldots, E_{k}$ of finite subsets of $I$, the intersection $X_{E_{1}} \cap \cdots \cap X_{E_{k}}$ contains $X_{E_{1} \cup \cdots \cup E_{k}}$. By Condition (iii), $X_{E}$ is non empty for any finite subset $E$ of $I$. Since $\widehat{A}$ is compact, it follows that

$$
\bigcap_{E} X_{E} \neq \emptyset,
$$

where $E$ runs over all finite subsets of $I$. Let $\chi \in \bigcap_{E} X_{E}$. It is easily checked that $\chi$ is $\Gamma$-faithful. 
Equivalence of (iv) and (v). Consider a finite subset $E$ of $I$. Recall that each $A_{i}$ is a finite dimensional vector space over a prime field $\mathbb{F}_{p_{i}}$, for a prime number $p_{i}$. For each prime $p$, denote by $V_{p}$ the direct sum of those $A_{i}$ with $i \in E$ which are vector spaces over $\mathbb{F}_{p}$, and denote by $P$ the set of primes $p$ such that $V_{p} \neq\{0\}$. We have $A_{E}=\bigoplus_{p \in P} V_{p}$. Since the $V_{p}$ 's are subgroups of $A_{E}$ of pairwise coprime orders, every subgroup $H$ of $A_{E}$ is a direct sum $\bigoplus_{p \in P}\left(H \cap V_{p}\right)$. The dual group $\widehat{V}_{p}$ of $V_{p}$ can be identified with the dual vector space $V_{p}^{*}$; as before, each subgroup $H^{*}$ of $\widehat{A}_{E}$ is a direct sum $\bigoplus_{p \in P}\left(H^{*} \cap V_{p}^{*}\right)$. It follows that, in order to prove the equivalence of (iv) and (v), we can assume that $P$ consists of a single element $p$. We can also assume that $\Gamma$ is a subgroup of $\operatorname{GL}\left(V_{p}\right)$.

Let $\mathbb{F}_{p}[\Gamma]$ denote the group algebra of $\Gamma$ over $\mathbb{F}_{p}$. Observe that $V_{p}$ is a semisimple $\mathbb{F}_{p}[\Gamma]$-module, since $V_{p}$ is a direct sum of minimal normal subgroups of $\Gamma$. (A module is semi-simple if it is a direct sum of simple modules; other authors use the terminology completely reducible.)

Under the identification of $\widehat{V}_{p}$ with $V_{p}^{*}$, the $\Gamma$-action on $\widehat{V}_{p}$ corresponds to the dual (or contragredient) action of $\Gamma$ on $V_{p}^{*}$. Observe that $V_{p}^{*}$ is a semi-simple $\mathbb{F}_{p}[\Gamma]$ module. Indeed, if $W$ is submodule of $V_{p}^{*}$, then its annihilator $W^{\perp}$ has a complement $Z$ in $V_{p}$ and $Z^{\perp}$ is a complement of $W$ in $V_{p}^{*}$ (compare with Lemma 6.2 in [Landr83]).

Observe also that there exists $x \in V_{p}$ such that $V_{p}$ is generated by the $\Gamma$-conjugacy class of $x$ (respectively, there exists $\chi \in \widehat{V}_{p}$ such that $\widehat{V}_{p}$ is generated by the $\Gamma$-orbit of $\chi$ ) if and only if $V_{p}$ (respectively $V_{p}^{*}$ ) is isomorphic, as $\mathbb{F}_{p}[\Gamma]$-module, to a quotient of the left regular module $\mathbb{F}_{p}[\Gamma]$. To conclude the proof, we show that $V_{p}$ is isomorphic to a quotient of $\mathbb{F}_{p}[\Gamma]$ if and only if $V_{p}^{*}$ is isomorphic to a quotient of $\mathbb{F}_{p}[\Gamma]$.

We first show that every semi-simple submodule of $\mathbb{F}_{p}[\Gamma]$ is isomorphic to a quotient of $\mathbb{F}_{p}[\Gamma]$. Indeed, let $\mathbb{F}_{p}[\Gamma]=\bigoplus_{j \in J} P_{j}$ be a direct sum decomposition of $\mathbb{F}_{p}[\Gamma]$ into indecomposable submodules $P_{j}$. Every $P_{j}$ contains a unique simple module $S_{j}$. Moreover, $S_{j}$ is isomorphic to a quotient of $P_{j}$ and $M=\bigoplus_{j \in J} S_{j}$ is the sum of all simple submodules of $\mathbb{F}_{p}[\Gamma]$. For the standard facts on representation theory of finite groups, see for example [Landr83], in particular Theorem 6.8. Let $N$ be a semi-simple submodule of $\mathbb{F}_{p}[\Gamma]$. Then $N$ is a submodule of $M$ and is therefore isomorphic to $\bigoplus_{j \in J^{\prime}} S_{j}$ for a subset $J^{\prime}$ of $J$. Hence, $N$ is isomorphic to a quotient of $\bigoplus_{j \in J^{\prime}} P_{j}$. Since $\bigoplus_{j \in J^{\prime}} P_{j}$ is a direct summand of $\mathbb{F}_{p}[\Gamma]$, it follows that $N$ is isomorphic to a quotient of $\mathbb{F}_{p}[\Gamma]$ and this proves our claim.

Assume that $V_{p}$ is isomorphic to a quotient of $\mathbb{F}_{p}[\Gamma]$. Then $V_{p}^{*}$ is isomorphic to a submodule of $\mathbb{F}_{p}[\Gamma]^{*}$. Now, it is standard that $\mathbb{F}_{p}[\Gamma]^{*}$ is isomorphic to $\mathbb{F}_{p}[\Gamma]$ as a $\mathbb{F}_{p}[\Gamma]$-module (see [Landr83, Theorem 6.3]). Hence, $V_{p}^{*}$ is isomorphic to a submodule of $\mathbb{F}_{p}[\Gamma]$. By what we have seen above, it follows that $V_{p}^{*}$ is isomorphic to a quotient of $\mathbb{F}_{p}[\Gamma]$. Similarly, if $V_{p}^{*}$ is isomorphic to a quotient of $\mathbb{F}_{p}[\Gamma]$, then $V_{p}$ is isomorphic to a quotient of $\mathbb{F}_{p}[\Gamma]$. 


\section{Finite and infinite dimensional representations}

Our proof of Theorem 4 uses the following elementary lemma, which is well known. To our surprise, we haven't been able to find a convenient reference.

Lemma 15. Let $\Omega$ a standard Borel space and $\mu$ a bounded positive measure on $\Omega$.

(i) Let $A$ be a separable $C^{*}$-algebra, $\underline{\pi}$ a representation of $A$, and

$$
\underline{\pi}=\int_{\Omega}^{\oplus} \underline{\pi}_{\omega} d \mu(\omega)
$$

a direct integral decomposition of $\underline{\pi}$ with respect to a measurable field $\omega \mapsto \underline{\pi}_{\omega}$ of representations of $A$. Then $\underline{\pi}_{\omega}$ is weakly contained in $\underline{\pi}$ for almost all $\omega$ in $\Omega$.

(ii) Let $\Gamma$ be a countable group, $\pi$ a representation of $\Gamma$, and

$$
\pi=\int_{\Omega}^{\oplus} \pi_{\omega} d \mu(\omega)
$$

a direct integral decomposition of $\pi$ with respect to a measurable field $\omega \mapsto \pi_{\omega}$ of representations of $\Gamma$. Then $\pi_{\omega}$ is weakly contained in $\pi$ for almost all $\omega \in \Omega$.

Proof. (i) By definition of "weak containment", we have to show that $\operatorname{ker}(\underline{\pi}) \subset$ $\operatorname{ker}\left(\underline{\pi}_{\omega}\right)$ for almost all $\omega \in \Omega$. Since $A$ is separable, so is $\operatorname{ker}(\underline{\pi})$, and we can choose in this kernel a countable dense subset, say $C$. For any $x \in A$, recall from the theory of direct integrals that $\|\underline{\pi}(x)\|$ is the essential supremum (on $\omega \in \Omega$ ) of the norms $\left\|\underline{\pi}_{\omega}(x)\right\|$, so that $\left\|\underline{\pi}_{\omega}(x)\right\| \leq\|\underline{\pi}(x)\|$ for almost all $\omega \in \Omega$; in particular, any $x \in C$ is in $\operatorname{ker}\left(\pi_{\omega}\right)$ for almost all $\omega \in \Omega$. Since $C$ is countable, we have also $C \subset \operatorname{ker}\left(\pi_{\omega}\right)$ for almost all $\omega \in \Omega$, and this implies the announced conclusion.

(ii) Any representation $\pi$ of $\Gamma$ corresponds to a representation $\pi$ of the maximal $\mathrm{C}^{*}$-algebra $A=C_{\max }^{*}(\Gamma)$ of the group. For two representations $\pi_{1}, \pi_{2}$ of the group, $\pi_{1}$ is weakly contained in $\pi_{2}$ if and only if $\operatorname{ker}\left(\underline{\pi}_{2}\right) \subset \operatorname{ker}\left(\underline{\pi}_{1}\right)$; moreover, a direct integral decomposition $\pi=\int_{\Omega}^{\oplus} \pi_{\omega} d \mu(\omega)$ at the level of $\Gamma$ corresponds to a direct integral decomposition $\underline{\pi}=\int_{\Omega}^{\oplus} \underline{\pi}_{\omega} d \mu(\omega)$ at the level of $C_{\max }^{*}(\Gamma)$, with the same space $\Omega$ and the same measure $\mu$. Thus (ii) is a consequence of (i).

[More generally, both (ii) and its proof hold verbatim for representations of separable locally compact groups.]

To prove Theorem 4, it is clearly enough to show that Conditions (i) and (ii) there are equivalent. The implication (i) $\Longrightarrow$ (ii) is a straightforward consequence of [Thoma64, Korollar 1], according to which every irreducible representation of a virtually abelian group is finite dimensional. 
End of proof of Theorem 4, namely of (ii) $\Longrightarrow$ (i). We assume that $\Gamma$ has Property (ii), and we split the proof in two cases.

Assume first that $\Gamma$ is not amenable. Let $\sigma$ be a $\Gamma$-faithful irreducible representation of $M S(\Gamma)$; set $\pi=\operatorname{Ind}_{M S(\Gamma)}^{\Gamma} \sigma$. By Lemmas 10 and 15, some (in fact almost every) irreducible representation $\pi_{0}$ which occurs in some direct integral decomposition of $\pi$ is faithful and is weakly contained in $\pi$. As $M S(\Gamma)$ is amenable, $\pi$ is weakly contained in the left regular representation of $\Gamma$, and therefore the same holds for $\pi_{0}$. As $\Gamma$ is not amenable, $\pi_{0}$ cannot be finite dimensional, so that $\Gamma$ has Property (i).

Assume now that $\Gamma$ is amenable. Assume furthermore, by contradiction, that $\Gamma$ does not have Property (i). Then $\Gamma$ has a finite dimensional faithful irreducible representation, by the first part of (ii). In particular, $\Gamma$ can be viewed as a subgroup of the compact unitary group $\mathcal{U}(n)$, for some integer $n \geq 1$. By Tits' alternative [Tits79], there exists in $\Gamma$ a soluble subgroup $\Delta$ of finite index. Let $R$ denote the closure of $\Delta$ in $U(n)$ and let $R^{0}$ denote its connected component; then $R^{0}$ is of finite index in $R$ (because $R$ is a compact Lie group, see for example [Helga62, Chapter II, Theorem 2.3]) and an abelian group (because a connected compact group is soluble if and only if it is abelian, see for example [Bourb82, Appendice I]). Thus $\Delta \cap R^{0}$ is an abelian subgroup of finite index in $\Gamma$; but this contradicts the hypothesis that $\Gamma$ is not virtually abelian, and this ends the proof.

Proposition 16. Let $\Gamma$ be a countable group.

(i) If there exists a factor $M$ and an injective homomorphism $\pi: \Gamma \rightarrow \mathcal{U}(M)$ such that $\pi(\Gamma)^{\prime \prime}=M$, then $\Gamma$ is irreducibly represented.

(ii) If $\Gamma$ is irreducibly represented, then there exists a factor $M=\mathscr{L}(\mathscr{H})$ of type I and a faithful representation $\pi: \Gamma \rightarrow \mathcal{U}(\mathscr{H})$ such that $\pi(\Gamma)^{\prime \prime}=\mathscr{L}(\mathscr{H})$.

Proof. Let $\pi$ be as in (i). If $M$ is an algebra of operators on some Hilbert space $\mathcal{K}$, then $\pi$ is in particular a factorial representation of $\Gamma$ in $\mathcal{K}$. It corresponds to a $\mathrm{C}^{*}$-representation, say $\underline{\pi}: C_{\max }^{*}(\Gamma) \rightarrow \mathscr{L}(\mathcal{K})$. By a result of Dixmier (Corollary 3, page 100 of [Dix60]), there exists an irreducible representation $\rho$ of $C_{\max }^{*}(\Gamma)$ such that $\underline{\pi}$ and $\rho$ have the same kernel. The restriction $\rho$ of $\rho$ to $\Gamma$ is therefore a faithful irreducible representation.

In view of Schur's lemma, (ii) is nothing but a reformulation of the definition of "irreducibly represented".

Corollary 5 is a straightforward consequence of Theorem 4 and Proposition 16.

Short of knowing how to answer the questions which follow Corollary 5, let us record the following elementary remark.

Observation. If $\Gamma$ is a countable group which has a finite dimensional faithful irreducible representation, then $M H(\Gamma)$ is a finite group. 
Proof. Consider the following properties of a group $\Gamma$ :

(a) $\Gamma$ has a finite dimensional faithful irreducible representation;

(b) $M S(\Gamma)$ has a finite dimensional $\Gamma$-faithful irreducible representation;

(c) $M A(\Gamma)$ has a $\Gamma$-faithful character and $M H(\Gamma)$ has a finite dimensional faithful irreducible representation;

(d) $M A(\Gamma)$ has a $\Gamma$-faithful character and $M H(\Gamma)$ is a finite group.

Property (a) implies Property (b) by Lemma 9, Properties (b) and (c) are equivalent because $M S(\Gamma)=M A(\Gamma) \oplus M H(\Gamma)$, and Properties (c) and (d) are equivalent because $M H(\Gamma)$ is a direct sum of finite simple groups.

[Observe that, however, Property (b) does not imply Property (a): if $\Gamma$ is an icc group which does not have any finite dimensional faithful representation, for example the group of permutations of finite support of $\mathbb{Z}$, then $\Gamma$ has Property (b) since $M S(\Gamma)=\{e\}$, but does not have Property (a).]

About Conditions (ii) and (iv) of Theorem 4, let us moreover recall the following facts. For countable groups, and more generally for separable locally compact groups and for separable $\mathrm{C}^{*}$-algebras, there is a notion of being of type $I$, defined in terms of the von Neumann algebras generated by the images of appropriate representations. It is then a theorem of Thoma that a countable group is of type I if and only if it is virtually abelian, if and only if all its irreducible representations are finite dimensional. See [Thoma64] and [Glimm61].

\section{Remarks}

5.1. Minisocles, socles, and examples. The socle of a group $\Gamma$ is the subgroup $S(\Gamma)$ generated by the union of the minimal normal subgroups (finite or infinite). Here are some examples of socles and minisocles.

(I) For a prime $p$ and an integer $n \geq 1$, the socle of the finite cyclic group $\mathbb{Z} / p^{n} \mathbb{Z}$ is isomorphic to $\mathbb{Z} / p \mathbb{Z}$. The socle of the finite $\operatorname{symmetric}$ group $\operatorname{Sym}(n)$ is the corresponding alternating group if $n=3$ or $n \geq 5$, and the Vierergruppe if $n=4$.

If $\Gamma$ is a 2-transitive subgroup of $\operatorname{Sym}(n)$, then $S(\Gamma)$ is either of the form $\left(\mathbb{F}_{p}\right)^{m}$ or a finite simple group. More generally and more precisely, if $\Gamma$ is a primitive subgroup of $\operatorname{Sym}(n)$, the O'Nan-Scott Theorem (1980) provides detailed information on the socle of $\Gamma$; in particular, $S(\Gamma) \approx S^{m}$ for some finite simple group $S$ and some integer $m$. See for example Chapter 4 in [DixMo96].

(II) Free abelian groups $\mathbb{Z}^{n}, n \geq 1$, and nonabelian free groups have socles reduced to one element. 
(III) For $n \geq 3$, the socle of $\mathrm{SL}_{n}(\mathbb{Z})$ is reduced to one element or is of order two, if $n$ is odd or even respectively (because any noncentral normal subgroup of $\mathrm{SL}_{n}(\mathbb{Z})$ contains a congruence subgroup, and consequently is never minimal).

(IV) Let $\Gamma$ be a lattice in a connected semisimple Lie group $G$ with finite center $Z(G)$ and without compact factor. It is an easy consequence of the Borel density theorem that, if the centre of $\Gamma$ is $\{e\}$, then $\Gamma$ is icc, so that $M S(\Gamma)=\{e\}$; more generally, $M S(\Gamma)=\Gamma \cap Z(G)$.

The minisocle of a just infinite group is reduced to one element (by definition). In particular, the minisocle of the Grigorchuk group is reduced to one element.

(V) If $\Gamma$ is a direct sum of a family of infinite simple groups, then $M S(\Gamma)=\{e\}$ and $S(\Gamma)=\Gamma$. If $\Gamma$ is a direct sum of a family of finite simple groups, then $M S(\Gamma)=\Gamma$.

(VI) The socle of a nilpotent group $\Gamma$ is contained in the centre $Z(\Gamma)$ of $\Gamma$, because $N \cap Z(\Gamma) \neq\{e\}$ for any normal subgroup $N \neq\{e\}$ of $\Gamma$.

(VII) Let $\Gamma$ be an abelian torsion-free group with cardinal strictly larger than that of the real numbers, for example a direct product of copies of $\mathbb{Z}$ indexed by $\mathbb{R}$. Then $M S(\Gamma)$ is reduced to one element, and $\Gamma$ does not have any faithful character, so that the equivalences of Theorem 2 do not hold for $\Gamma$.

(VIII) Let $H$ be a group, $p$ a prime number, $U$ a vector space over the prime field with $p$ elements, $\pi: H \rightarrow \mathrm{GL}(U)$ a faithful representation which is semi-simple (namely which is a direct sum of irreducible representations), and $\Gamma=H \ltimes U$ the corresponding semi-direct product. Then $U$ is the socle of $\Gamma$.

Indeed, let $N$ a minimal normal subgroup of $\Gamma$. If $N \cap U \neq\{0\}$, then $N \subset U$, and moreover $N$ is a $H$-invariant subspace of $U$ which is irreducible, by minimality; these $N$ 's generate $U$. If one had $N \cap U=\{0\}$, then $N$ and $U$ would commute (being two normal subgroups of $\Gamma$ ), so that $N$ would act trivially on $U$, and this is ruled out by the faithfulness of $\pi$.

Let $U$ be of the form $U=\left(\bigoplus_{i \in I} V_{i}\right) \oplus\left(\bigoplus_{j \in J} W_{j}\right)$, with each $V_{i}$ a $H$-invariant irreducible finite-dimensional subspace of $U$, and each $W_{j}$ a $H$-invariant irreducible infinite-dimensional subspace of $U$. Then the mini-socle of $\Gamma$ is $\bigoplus_{i \in I} V_{i}$.

The construction carries over to the case where each $V_{i}$ and $W_{j}$ is a vector space over a prime field of which the number of elements depends on $i$ and $j$.

5.2. Minisocles, FC-kernels, and $\mathbf{P}$. Hall's theorems. The $F C$-kernel of a group $\Gamma$ is the subset $\Gamma_{\mathrm{FC}}$ of $\Gamma$ of elements which have a finite conjugacy class. It is a characteristic subgroup of $\Gamma$.

The periodic FC-kernel of $\Gamma$ is the subset $\Gamma_{\mathrm{FC}}^{\mathrm{per}}$ of $\Gamma_{\mathrm{FC}}$ of elements of finite order. It is also a subgroup of $\Gamma$, indeed a locally finite subgroup (Dicman's Lemma, see 
e.g. [Tomki84]). It follows from the definitions and from Dicman's Lemma that

$$
M S(\Gamma) \subset \Gamma_{\mathrm{FC}}^{\mathrm{per}}
$$

(the inclusion can be strict, as it is for example the case if $\Gamma$ is cyclic of order four).

Any subgroup of a restricted direct product of finite groups is a periodic FCgroup which is residually finite, and any quotient of a periodic FC-group is a periodic FC-group. For countable groups, Philip Hall has established in 1959 the converse implications:

any countable periodic FC-group which is residually finite can be embedded in a restricted direct product of finite groups, and any countable periodic FC-group is isomorphic to a quotient of a subgroup of a restricted direct product of finite groups

(Theorems 2.5 and 3.2 in [Tomki84]).

A hoof of a group $\Gamma$ is a foot of a foot. Thus, with the notation of Proposition 1, the subgroups $\mathbb{F}_{p}$ and $S_{1}$ are hooves of $\Gamma$.

Let $\Gamma$ be a group which has a finite Jordan-Hölder sequence (for example a finite group); if a simple group $S$ is a foot of $\Gamma$, then $S$ is isomorphic to a quotient of some Jordan-Hölder sequence of $\Gamma$ (Bourbaki, Algèbre, nouvelle édition, 1970, chap. I, $\S 4$, no 7). But the converse does not hold: the group of order 3 is a simple quotient of a Jordan-Hölder sequence of the alternating group Alt(4) of order 12, but Alt(4) has a unique foot which is the Vierergruppe, of order 4.

5.3. Recall of a theorem of Gelfand and Raikov. Recall the following basic result of the theory of group representations, due to Gelfand and Raikov (see [GelRa42], as well as Corollary 13.6.6 in [Dix69C* ]):

for any $\gamma \in \Gamma, \gamma \neq e$, there exists an irreducible representation $\pi_{\gamma}$ such that $\pi_{\gamma}(\gamma) \neq \pi_{\gamma}(e)$.

This holds for any group $\Gamma$, countable or not; indeed, this holds for any locally compact group, with $\pi_{\gamma}$ a continuous representation. There are two main ingredients of the proof: the group has a faithful representation which is the left-regular representation, and any representation has some description in terms of irreducible representations (via functions of positive types and a theorem of the Krein-Milman type).

For a countable group $\Gamma$ which has the properties (ii) to (v) of Theorem 2, we have shown that $\pi_{\gamma}$ can be chosen independently of $\gamma$.

5.4. Recall on tensor powers of faithful representations. Let $\Gamma$ be a group and let $\pi$ be a faithful representation of $\Gamma$. For integers $m, n \geq 0$, consider the tensor power $\pi_{m, n}=\pi^{\otimes m} \otimes \bar{\pi}^{\otimes n}$, where $\bar{\pi}$ denotes the representation conjugate to $\pi$ and $\pi^{\otimes m}$ the tensor product of $m$ copies of $\pi$. Then the left regular representation of $\Gamma$ is weakly contained in the direct sum $\bigoplus_{m, n \geq 0} \pi_{m, n}$ (see Example 1.11 in [BeLaS92]). 
This is a generalisation, with weak containment replacing strong containment, of a well-known fact about finite groups (and compact groups, see [Cheva46], Chapter VI, $\S$ VII, Proposition 3). Thus, if, in addition, $\Gamma$ is amenable, then every representation of $\Gamma$ is weakly contained in $\bigoplus_{m, n \geq 0} \pi_{m, n}$. (All this carries over to locally compact groups.)

For a countable group $\Gamma$ which has the properties (ii) to (v) of Theorem 2, the representation $\pi$ can be chosen to be faithful and irreducible.

5.5. Primitive group $\mathbf{C}^{*}$-algebras. Denote by $C_{\text {red }}^{*}(\Gamma)$ the reduced $C^{*}$-algebra, and as above by $C_{\max }^{*}(\Gamma)$ the maximal $C^{*}$-algebra of a group $\Gamma$. A representation of either one of these algebras is irreducible if and only if its restriction to $\Gamma$ is irreducible. It follows that, if one of $C_{\mathrm{red}}^{*}(\Gamma), C_{\max }^{*}(\Gamma)$ is primitive, then $\Gamma$ is irreducibly represented.

Many examples of countable groups are known for which $C_{\mathrm{red}}^{*}(\Gamma)$ is simple [Harpe07], and a fortiori primitive. These groups are in particular irreducibly represented. Concerning the properties of $\Gamma$ and $C_{\text {red }}^{*}(\Gamma)$, consider the three following conditions:

(NF) $\Gamma$ does not have any finite normal subgroup besides $\{e\}$;

(NA) $\Gamma$ does not have any amenable normal subgroup besides $\{e\}$;

$\left(\mathrm{C}^{*} \mathrm{~S}\right) C_{\text {red }}^{*}(\Gamma)$ is simple.

It is straightforward that (NF) is a rephrasing of the condition $M S(\Gamma)=\{e\}$, and that it follows from (NA). It is elementary to check that (NA) follows from $\left(\mathrm{C}^{*} \mathrm{~S}\right)$, but we recall that it is not known whether the converse holds (see [BekHa00] and [Harpe07]).

If $\Gamma$ is amenable, the $\mathrm{C}^{*}$-algebras $C_{\text {red }}^{*}(\Gamma)$ and $C_{\max }^{*}(\Gamma)$ are isomorphic. They are primitive if and only if $\Gamma$ is icc [Murph03].

If $\Gamma$ is a nonabelian free group, it is a result of Yoshiwaza that $C_{\max }^{*}(\Gamma)$ is primitive (see [Yoshi51], as well as [Choi80]). See the discussion around Problem 25 in [Harpe07].

\section{A generalisation of Theorem 2}

Consider a countable group $\Gamma$ and a subgroup $G$ of the automorphism group of $\Gamma$ which contains all inner automorphisms. There is an obvious notion of $G$-faithful representation, which coincides with that of $\Gamma$-faithful representation in case $G$ coincides with the group of inner automorphisms. Observe that $M S(\Gamma), M A(\Gamma)$, and $M H(\Gamma)$ are $G$-invariant subgroups of $\Gamma$, since all three are characteristic.

We define a $G$-foot to be a minimal $G$-invariant subgroup of $\Gamma$. Let $\mathcal{F}_{\Gamma}^{G}$ denote the set of finite $G$-feet of $\Gamma$; it is the union of the set $\mathcal{A}_{\Gamma}^{G}$ of abelian finite $G$-feet and 
of its complement $\mathscr{H}_{\Gamma}^{G}$. The $G$-minisocle of $\Gamma$ is the subgroup $M S^{G}(\Gamma)$ generated by its $G$-feet, and we have as in Section 1 subgroups $M A^{G}(\Gamma)$ and $M H^{G}(\Gamma)$.

Proposition 17. Let $\Gamma$ and $G$ be as above.

(i) Each $B \in \mathcal{A}_{\Gamma}^{G}$ is isomorphic to $\left(\mathbb{F}_{p}\right)^{n}$ for some prime $p$ and some positive integer $n$ (depending on $B$ ).

(ii) There exists a subset $\left\{B_{i}\right\}_{i \in I}$ of $\mathcal{A}_{\Gamma}^{G}$ such that $M A^{G}(\Gamma)=\bigoplus_{i \in I} B_{i}$. In particular, the group $M A^{G}(\Gamma)$ is abelian.

(iii) For each $H \in \mathscr{H}_{\Gamma}^{G}$, the feet $S_{1}, \ldots, S_{k}$ of $H$ are conjugate under $G$, and simple. Moreover $H=S_{1} \oplus \cdots \oplus S_{k}$.

(iv) We have $M H^{G}(\Gamma)=\bigoplus_{H \in \mathscr{H}_{\Gamma}^{G}} H$.

(v) We have $M S^{G}(\Gamma)=M A^{G}(\Gamma) \oplus M H^{G}(\Gamma)$.

Theorem 18. Let $\Gamma, G$, and $M A^{G}(\Gamma)=\bigoplus_{i \in I} B_{i}$ be as above. The following properties are equivalent:

(i) $\Gamma$ has a representation which is irreducible and $G$-faithful;

(ii) $M A(\Gamma)$ has a $G$-faithful character;

(ii') $M A^{G}(\Gamma)$ has a $G$-faithful character;

(iii) $M S(\Gamma)$ has a $G$-faithful irreducible representation;

(iii') $M S^{G}(\Gamma)$ has a $G$-faithful irreducible representation;

(iv) for every finite subset $E$ of $I$, there exists an element $x_{E}$ in $M A_{E}^{G}(\Gamma) \doteqdot$ $\bigoplus_{i \in E} B_{i}$ such that the $G$-orbit of $x_{E}$ generates $M A_{E}^{G}(\Gamma)$.

In particular, a countable group $\Gamma$ has a $G$-faithful irreducible representation as soon as $M A^{G}(\Gamma)=\{e\}$, a fortiori as soon as $M S^{G}(\Gamma)=\{e\}$.

For example, let $\Gamma=\bigoplus_{i \in \mathbb{N}} A_{i}$ be a countable infinite direct sum of groups $A_{i}$ indexed by the natural numbers, each of them isomorphic to a given finite cyclic group, and let $G$ be the group of permutations of $\mathbb{N}$, identified in the natural way to a group of automorphisms of $\Gamma$. Then $\Gamma$ is irreducibly underrepresented, but has a $G$-faithful irreducible character, for example the projection onto $A_{1}$ followed by the natural isomorphisms of $A_{1}$ with the appropriate group of roots of unity.

Proposition 17 and Theorem 18 can be proved by essentially the same arguments as in Sections 2 and 3.

Lemmas 9 and 10 should be reformulated for a $G$-invariant subgroup $N$ of $\Gamma$; in the new Lemma 9, the $G$-faithfulness of $\pi$ implies that $\sigma_{\omega}$ is $G$-faithful for almost all $\omega \in \Omega$; in the new Lemma 10, if we assume that $\sigma$ is $G$-faithful and that there does not exist any finite $G$-foot $M$ such that $M \cap N=\{e\}$, then $\pi_{\omega}$ is $G$-faithful for 
almost all $\omega \in \Omega$. In the new Lemma 13, the groups $N, A, S$ should be $G$-invariant, but $S_{i}$ normal (not necessarily $G$-invariant) and simple (not necessarily $G$-simple); the conclusion is that $N$ has a $G$-faithful irreducible representation if and only if $A$ has a $G$-faithful character. In the new Lemma 14, both $A$ and the $A_{i}$ should be $G$-invariant, and $\chi^{\Gamma}$ should be replaced by $\chi^{G}$. The other (minor) modifications, as well as the formulation of one more claim analogous to Claim (v) of Theorem 2, are left to the reader.

\section{References}

[BekHa00] B. Bekka and P. de la Harpe, Groups with simple reduced C* -algebras. Expositiones Math. 18 (2000), 215-230. Zbl 0966.46032 MR 1763888

[BeLaS92] B. Bekka, A. Lau, and G. Schlichting, On invariant subalgebras of the FourierStiletjes algebra of a locally compact groups. Math. Ann. 294 (1992), 513-522. Zbl 0787.22005 MR 1188135

[Bourb82] N. Bourbaki, Groupes et algèbres de Lie. Chapitre 9, Masson (1982). Zbl 0505.22006 MR 0682756

[Burns11] W. Burnside, The theory of groups of finite order. 2nd Edition, Cambridge University Press, Cambridge 1911.

[Cheva46] C. Chevalley Theory of Lie groups I. Princeton University Press, Princeton, NJ, 1946. Zbl 0063.00842 MR 0082628

[Choi80] M.-D. Choi, The full C*-algebra of the free group of two generators. Pacific J. Math. 87 (1980), 41-48. Zbl 0463.46047 MR 0590864

[Dix60] J. Dixmier, Sur les C*-algèbres. Bull. Soc. Math. France 88 (1960), 95-112. Zbl 0124.32403 MR 0121674

[Dix69C*] J. Dixmier, Les $C^{*}$-algèbres et leurs représentations. Gauthier-Villars, Paris 1969. Zbl 0174.18601 MR 0246136

[Dix69vN] J. Dixmier, Les algèbres d'opérateurs dans l'espace hilbertien (algèbres de von Neumann). Deuxième édition, Gauthier-Villars, Paris 1969. Zbl 0175.43801 MR 0352996

[DixMo96] J. D. Dixon and B. Mortimer, Permutation groups. Grad. Texts in Math. 163, Springer-Verlag, New York 1996. Zbl 0951.20001 MR 1409812

[Dixon71] J. D. Dixon, The structure of linear groups. Van Nostrand, 1971 Zbl 0232.20079

[Gasch54] W. Gaschütz, Endliche Gruppen mit treuen absolut-irreduziblen Darstellungen. Math. Nach. 12 (1954), 253-255 Zbl 0056.25703 MR 0067115

[GelG108] T. Gelander and Y. Glasner, Countable primitive groups. Geom. Funct. Anal. 17 (2008), 1479-1523. Zbl 1138.20005 MR 2377495

[GelRa42] I. M. Gelfand and D. A. Rajkov, Irreducible unitary representations of locally bicompact groups. Mat. Sb. 13 (55) (1942), 301-316 [= I. M. Gelfand, Collected papers, Volume II, pages 3-17]. Zbl 0063.01566 MR 0011308 
[GlaMo07] Y. Glasner and N. Monod, Amenable actions, free products, and a fixed point property. Bull. London Math. Soc. 39 (2007), 138-150. Zbl 05139551 MR 2303529

[Glimm61] J. Glimm, Type IC*-algebras. Annals of Math. 73 (1961), 572-612.Zbl 0152.33002 MR 0124756

[Guich66] A. Guichardet, Produits tensoriels infinis et représentations des relations d'anticommutation. Ann. Sci. Ec. Normale Sup. 83 (1966), 1-52. Zbl 0154.38905 MR 0205097

[Harpe07] P. de la Harpe, On simplicity of reduced C* -algebras of groups. Bull. London Math. Soc. 39 (2007), 1-26. Zbl 05139536 MR 2303514

[Helga62] S. Helgason, Differential geometry and symmetric spaces. Academic Press, New York 1962. Zbl 0111.18101 MR 0145455

[Hupp98] B. Huppert, Character theory of finite groups. Walter de Gruyter, Berlin 1998. Zbl 0932.20007 MR 1645304

[Landr83] P. Landrock, Finite group algebras and their modules. London Math. Soc. Lecture Notes Ser. 84, Cambridge University Press, Cambridge 1983. Zbl 0523.20001 MR 0737910

[Murph03] G. J. Murphy, Primitivity conditions for full group C*-algebras. Bull. London Math. Soc. 35 (2003), 697-305. Zbl 1060.46041 MR 1989500

[Pálfy79] P. P. Pálfy, On faithful irreducible representations of finite groups. Studia Sci. Math. Hungar. 14 (1979), 95-98. Zbl 0426.20005 MR 0645518

[Remak30] R. Remak, Über minimale invariante Untergruppen in der Theorie der endlichen Gruppen. J. reine angew. Math. 162 (1930), 1-16. JFM 56.0128.08

[ROIV] F. J. Murray and J. von Neumann, On rings of operators, IV. Annals of Math. 44 (1943), 716-808. Zbl 0060.26903 MR 0009096

[Thoma64] E. Thoma, Über unitäre Darstellungen abzälbarer, diskreter Gruppen. Math. Ann. 153 (1964), 111-138. Zbl 0136.11603 MR 0160118

[Tits79] J. Tits, Free Subgroups in Linear Groups. J. of Algebra 20 (1979), 250-270. Zbl 0236.20032 MR 0286898

[Tomki84] M. J. Tomkinson, FC-groups. Pitman, Boston, MA, 1984. Zbl 0547.20031 MR 0742777

[Tushe93] A. V. Tushev, On exact irreducible representations of locally normal groups. Ukrainian Math. J. 4512 (1993), 1900-1906. Zbl 0830.20014 MR 1357142

[Yoshi51] H. Yoshizawa, Some remarks on unitary representations of the free group. Osaka Math. J. 3 (1951), 55-63. Zbl 0045.30103 MR 0041854

Received December 7, 2006

Bachir Bekka, UFR Mathématiques, Université de Rennes 1, Campus Beaulieu, 35042 Rennes Cedex

E-mail: bachir.bekka@univ-rennes1.fr

Pierre de la Harpe, Section de Mathématiques, Université de Genève, C.P. 64,

1211 Genève 4, Switzerland

E-mail: Pierre.delaHarpe@unige.ch 